Research Paper

\title{
Pre-treatment high body mass index is associated with poor survival in Asian premenopausal women with localized breast cancer
}

\author{
Yung-Chang Lin, $\mathrm{MD}^{1,3}{ }^{\varpi}$; Hsiao-Hsiang Cheng, MD²; Shin-Cheh Chen, MD; Wen-Chi Shen, MD; Yi-Ting \\ Huang, MD \\ 1. Division of Hematology/Oncology, Department of Internal Medicine, Chang Gung Memorial Hospital, Linkou Branch, Tao-Yuan, Taiwan. \\ 2. Kou Foundation Sun Yat-Sen Cancer Center, Taipei, Taiwan. \\ 3. School of Medicine, Chang Gung University, Tao-Yuan, Taiwan. \\ 4. Department of General Surgery, Chang Gung Memorial Hospital, Linkou Branch, Tao-Yuan, Taiwan. \\ 5. Department of Radiotherapy, Chang Gung Memorial Hospital, Linkou Branch, Tao-Yuan, Taiwan.
}

$\square$ Corresponding author: Yung-Chang Lin, MD, Division of Hematology/Oncology, Department of Internal Medicine, Chang Gung Memorial Hospital, Linkou Branch. Address: 5, Fu-Hsing Street, Kuei ShanTownship, Taoyuan City, Taiwan R.O.C. County code 333. Tel: +886-3-3281200, ext. 8825; Fax: +886-3-3283973; E-mail: yclinof@adm.cgmh.org.tw.

(c) The author(s). This is an open access article distributed under the terms of the Creative Commons Attribution License (https://creativecommons.org/licenses/by/4.0/). See http://ivyspring.com/terms for full terms and conditions.

Received: 2021.02.07; Accepted: 2021.05.19; Published: 2021.05.27

\begin{abstract}
Background: Obesity is associated with poor prognosis in breast cancer patients. This study aimed to evaluate the effect of obesity measured by body mass index (BMI) on survival of Taiwanese breast cancer patients in a single institution.

Methods: We observed 5000 patients who were diagnosed with stage I-III breast cancer between 1990 and 2005. Information on BMI at diagnosis, and clinical follow-up for disease recurrence and death, up to 20 years post-diagnosis were available. BMI (in $\mathrm{kg} / \mathrm{m}^{2}$ ) categories included normal weight $(\mathrm{BMl}<24)$, overweight $(24 \leq \mathrm{BMI}<27)$, and obesity $(\mathrm{BMI} \geq 27)$, according to recommendations from the Bureau of Health Promotion of Taiwan. The role of BMI and other known prognostic factors for patient survival were evaluated in this patient cohort.

Results: Obesity was associated with advanced stage, higher nuclear grade, and higher percentages of estrogen receptor (ER) positive. The median age of patients with a higher BMI was greater than the median age of patients with a lower BMI. Obesity was an independent prognostic factor of overall survival (OS) $(P<0.001)$, but not disease-free survival (DFS) $(P=0.067)$. We subsequently analyzed the impact of age-stratified BMI (age $<50$ and age $\geq 50$ years) to ameliorate the impact of age bias. Following subset analyses, obesity correlated with shorter DFS $(P=0.004)$ and OS $(P=0.009)$ only in women $<50$ years of age. Multivariate analysis revealed that $\mathrm{BMI}$ was an independent prognostic factor for both DFS and OS in this group of patients. Subset analysis revealed that in women $<50$ years old, the impact of BMI on survival was associated with higher stage, ER negativity.

Conclusion: $\mathrm{BMI}$ is an independent prognostic factor of OS and DFS in breast cancer patients aged $<50$ years. Although the cause-effect relationship between obesity and survival is unclear, we recommend that weight control measures in young breast cancer survivors should be considered.
\end{abstract}

Key words: breast cancer, obesity, prognostic factor, body mass index

\section{Introduction}

Obesity is an emerging public health issue worldwide [1]. It is linked not only with type 2 diabetic mellitus and cardiovascular disease, but also with an increased risk of developing several cancer types [2, 3]. Obesity is an established risk factor for breast cancer, and is also associated with an increased risk of endometrial, kidney, colon, and esophageal cancer [4-6]. 
Sex hormone-associated cancers such as breast cancer are related to, at least in part, an elevated level of endogenous hormones [7]. Obesity may promote breast cancer progression through various mechanisms, including increased sex hormone levels in post-menopausal obese women [8-10]. Interestingly, meta-analyses have shown that obesity increases the risk of breast cancer in postmenopausal women, but decreases the risk in premenopausal women $[8,11]$. This suggests that the effect of obesity on breast cancer biology might vary in different contexts of hormonal status, hormonal therapy, or other unknown mechanisms [5, 12-16]. A meta-analysis focus on premenopausal from Asian patients found that obesity is associated with breast cancer, whether the result is associated with ethic difference was unknown [17]. In breast cancer, obesity also has been shown to be associated with poor overall survival (OS) and disease-free survival (DFS) [18-23], advanced stage at diagnosis [24], unfavorable tumor grade, and suboptimal local and systemic treatment results [19-21, 25, 26]. Obesity could affect chemotherapy efficacy, and is reported to be an independent prognostic factor for pathological complete response rate after neoadjuvant chemotherapy [27]. However, these studies are inconsistent. Some studies show that body mass index (BMI) is an independent factor affecting OS or DFS in breast cancer patients $[15,19,20,23,25,28-31]$, while some show that obesity is prognostic factor only in premenopausal women [23, 31, 32]. A meta-analysis found that the effect of obesity was greater in premenopausal women than in postmenopausal women, although the hazard ratio was statistically insignificant [20]. Inconsistencies in determining the effect of obesity on breast cancer prognosis might be attributable to several factors such as the study methodology, patient size and population, and duration of follow-up. Patient ethnicity might also be taken into consideration.

The prevalence of obesity is relatively lower in Asian women than in Caucasian women. The definition of obesity in the Taiwanese population is BMI of $\geq 27$ as most Taiwanese studies adopted [33, 34 ], rather than the commonly used cutoff value of 30 in Western countries [18]. This was based on a national nutrition and health survey conducted between 1993-1996 [35], it showed that for most BMI values, the prevalence of metabolic disorders were higher for Taiwanese than for Caucasians, and BMI 27.5 were the cutoffs with the highest predictive value [36]. A few studies have suggested that obesity, despite its low prevalence in Asian women, remains a risk factor of developing breast cancer [37]. In this work, we retrieved the data from institutional cancer registry since 1990, and medical records review. The diagnoses and management of the patients in this cohort were, generally, in accordance with the common worldwide guidelines of standards such as the National Comprehensive Cancer Network or St. Gallen consensus [38]. This allows us to analyze the effect of BMI on survival of Taiwanese breast cancer patients in a practice-oriented setting. Herein, we report the results of the analysis of the effect of BMI on survival of Taiwanese breast cancer patients, and the relationships between BMI and known prognostic factors.

\section{Patients and Methods}

Between 1990 and 2005, a total of 5000 female breast cancer patients who underwent breast cancer surgery were registered at a single institution. Information on patient demographics, tumor characteristics, and adjuvant or neo-adjuvant chemotherapy, radiotherapy, or hormonal therapy and disease status was collected retrospectively from medical records. Information regarding BMI at the time of diagnosis was available. Patients were followed up for recurrence and death for up to 20 years. The dates of death were confirmed by national vital statistics data provided by the Taiwanese Department of Health. The conduct of this study was in accordance of declaration of Helsinki and was approved by the Institutional Review Board.

\section{Statistical analysis}

Patient and tumor characteristics within different BMI subsets were analyzed. Continuous variables were analyzed with repeated measures of analysis of variance, and categorical variables were analyzed by the chi-square or Fisher's exact test. DFS was calculated from the date of primary surgery to the date of documented recurrence. Patients who died, or were lost to follow-up without documented evidence of freedom from recurrence, were censored at the date of death or the last patient visit. OS was calculated from the date of primary surgery to the date of death.

The OS and DFS were estimated according to the Kaplan-Meier method. Differences in survival rates were calculated using the log-rank test. Prognostic factors including: tumor size, nodal status, hormone receptor status, and Human Epidermal Growth Factor Receptor 2 (HER2) status, were analyzed according to the Cox regression model for OS and DFS. A $P$ value of $<0.05$ was considered significant. All statistics were calculated using the SPSS software package (SSPS Inc., Chicago, IL, USA). 


\section{Results}

Between 1990 and 2005, data from 5000 female breast cancer patients were compiled. Patient BMI data were obtained at diagnosis and ranged from 13.5 to 48.3 (mean $=24.26$ ). The definitions of obesity and other categories were based on BMI according to recommendations from the Bureau of Health Promotion of Taiwan: normal weight, BMI <24; overweight, $24 \leq \mathrm{BMI}<27$; and obesity, BMI $\geq 27$. The numbers of normal weight, overweight and obese patients were 2605 (52.1\%), 1300 (26\%), and 1095 $(21.9 \%)$, respectively. The median age (years) for the three groups was 44.7, 49.8, and 52.5, respectively $(P<0.001)$. Despite the BMI definition of obesity being much lower than the commonly used BMI criteria in Western countries, obese Taiwanese patients had larger tumors (median size: $2.78 \mathrm{~cm}, P<0.001$ ) and more advanced nodal statuses $(P=0.007)$, compared to normal weight and overweight patients. Tumors in obese women were associated with higher nuclear grades $(P<0.001)$ and higher percentages of positive ER $(P=0.011)$ and negative HER2 expression $(P=0.039)$. In summary, the clinic-pathological features in obese breast cancer patients were mixed, with both favorable and unfavorable factors. The treatments that patients received, however, did not differ between the leaner and heavier breast cancer patients. In order to eliminate any potential effect of patient age on these factors, we arbitrarily grouped this cohort into patients $<50$ and $\geq 50$ years old, to represent pre- and post-menopause status respectively. There was no accurate information regarding the patients' menopausal status at diagnosis. The patient demographics and tumor characteristics, divided by age group and BMI

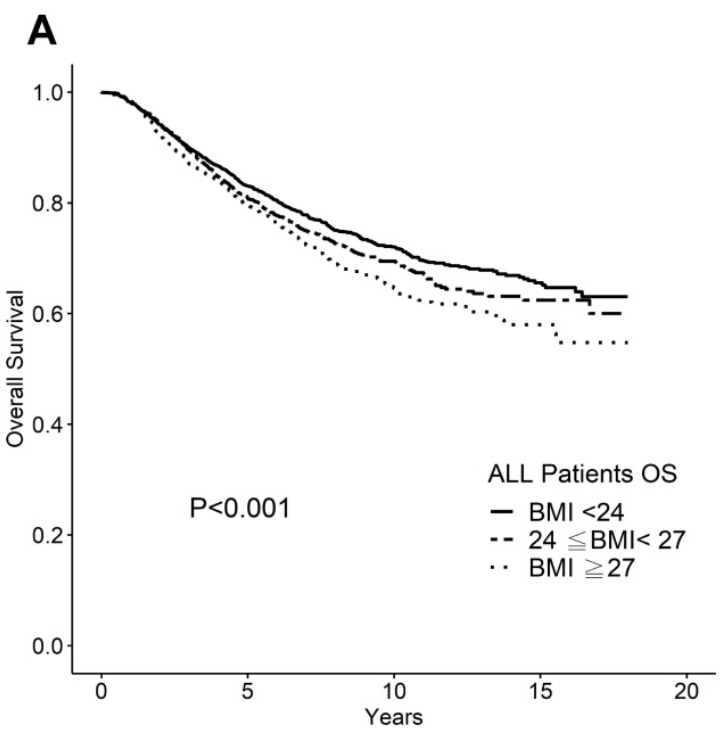

category, are summarized in Table 1 . The distribution pattern of these parameters was similar between the younger and older age groups. We found that nodal stage and nuclear grade were statistically more favorable in leaner patients within the premenopausal group. In post-menopausal women, a higher percentage of obese patients had hormonal receptor positive tumors; but there was no association between HER2 expression and BMI categories of either pre- or post-menopausal patients.

Higher BMI significantly associated with poor OS but not with DFS in a uni-variate analysis, as shown in Figure 1 . The 10 -year OS rates were $72 \%$, $69 \%$ and $66 \%$ for the normal weight, overweight, and obese BMI groups, respectively $(P<0.001)$. The 10 -year DFS rates were $66 \%, 65 \%$, and $63 \%$ for the normal weight, overweight, and obese groups, respectively $(P=0.067)$. When used BMI 30 as cutoff, the number of obese patients was only 433 (8.66\%), and the difference of 10-year DFS and OS were insignificant ( $p=0.289$, and 0.110, respectively). Even after multivariate analysis with the other known prognostic factors including tumor stage ( $\mathrm{T}$ stage), nodal stage, ER status, and HER2 status, BMI remained an independent prognostic factor for OS $(P$ $=0.007$; Table 2). Owing to our data retrieval limitations, we did not conduct a breast cancerspecific survival analysis. It is possible that the effect of BMI on OS could be attributed to co-morbidity or simply to age-related factors.

We further tested whether menopause status affects BMI as a prognostic factor, we analyzed the impact of BMI on survival by stratifying the patients into two age groups: $<50$ years and $\geq 50$ years. The clinicpathological features were compared between the two patient groups. The younger patient group

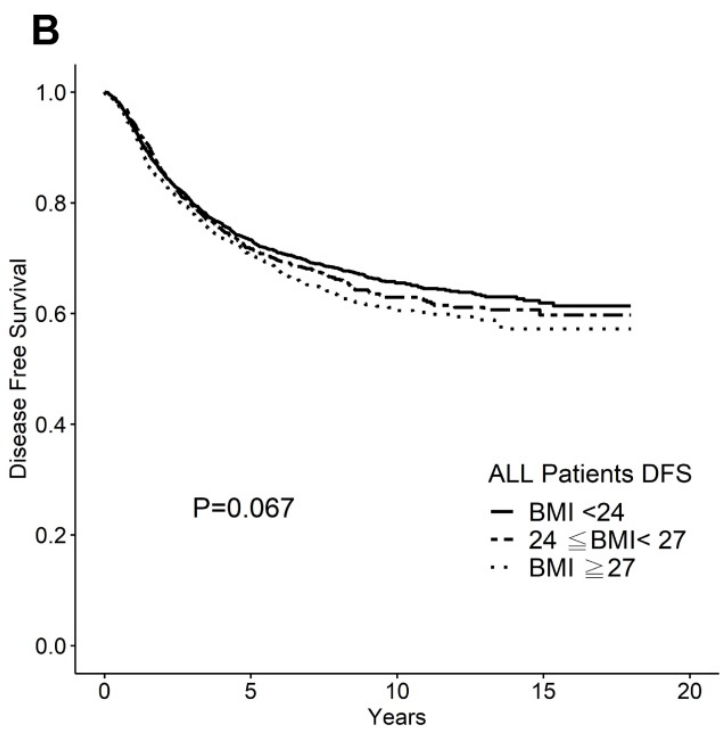

Figure 1. A. Overall survival according to BMI category for whole cohort. B. Disease-free survival according to BMI category for whole cohort. 
was associated with smaller tumors, lower nodal status, and low nuclear grades. In breast cancer patients aged $<50$ years, the 10 -year OS rates were $75 \%, 73 \%$, and $69 \%(P=0.009)$, and the 10 -year DFS rates were $67 \%, 65 \%$, and $60 \%(P=0.004)$, for normal weight (BMI <24), overweight $(24 \leq \mathrm{BMI}<27)$, and obese patients (BMI $\geq 27$ ), respectively. In contrast, for breast cancer patients aged $\geq 50$ years, neither the OS $(P=0.964)$ nor DFS $(P=0.965)$ were affected by BMI (Figure 2).

The significance of BMI as a prognostic factor for patients $<50$ years old was further demonstrated by multivariate analysis. Known prognostic factors such as tumor stage, nodal stage, as well as ER status along with HER2 status were selected for analysis in this cohort. The result indicated that BMI, $\mathrm{T}$ stage, nodal stage, ER status, and nuclear grade were independent prognostic factors for both DFS and OS (Table 3).

We subsequently analyzed the interactions between BMI and other established prognostic factors. Table 4 demonstrates the differences in 10-year survival rates for each T stage, nodal stage, and ER or HER2 status, stratified by BMI. For patients $<50$ years of age, the impact of BMI on survival was more significant in the node-positive and ER-negative patients (OS: $P=0.045$ and 0.001 , respectively; DFS: $P$ $=0.049$ and 0.001 , respectively).

Table 1. Demographic of Patients and Tumor Characteristics $(N=5000)$

\begin{tabular}{|c|c|c|c|c|c|c|c|}
\hline \multirow[t]{2}{*}{ Characteristic } & & \multicolumn{6}{|c|}{ BMI $\left(\mathrm{kg} / \mathrm{m}^{2}\right)$} \\
\hline & & \multicolumn{2}{|c|}{$<24(n=2605)$} & \multicolumn{2}{|c|}{$24 \leq \mathrm{BMI}<27(\mathrm{n}=1300)$} & \multicolumn{2}{|l|}{$\geq 27(n=1095)$} \\
\hline \multirow[t]{5}{*}{ Median age (mean) } & $P<0.001$ & $44(46)$ & & $49(51)$ & & $52(53)$ & \\
\hline & & \multicolumn{3}{|c|}{ Age $<50(n=2923)$} & \multicolumn{3}{|c|}{ Age $\geq 50(n=2077)$} \\
\hline & & \multicolumn{3}{|c|}{$B M I\left(k g / m^{2}\right)$} & \multicolumn{3}{|c|}{$B M I\left(k g / m^{2}\right)$} \\
\hline & & $<24$ & $24 \leq B M I<27$ & $\geq 27$ & $<24$ & $24 \leq B M I<27$ & $\geq 27$ \\
\hline & No. $(\%)$ & 1809 (61.9) & $661(22.6)$ & $453(15.5)$ & $796(38.3)$ & $639(30.8)$ & $642(30.9)$ \\
\hline \multirow[t]{2}{*}{ Mean tumor size $(\mathrm{cm})$} & & 2.5 & 2.57 & 2.89 & 2.48 & 2.56 & 2.70 \\
\hline & $P$ value & $0.043^{*}$ & $0.143^{* *}$ & & $0.024^{*}$ & $0.662^{* * *}$ & \\
\hline \multirow[t]{6}{*}{$\mathrm{T}(\%)$} & $\mathrm{T} 1$ & 748 (41.93) & $272(41.1)$ & $154(34)$ & $296(37.2)$ & $243(38.1)$ & $221(34.4)$ \\
\hline & $\mathrm{T} 2$ & $910(50.3)$ & $335(50.7)$ & $256(56.6)$ & $446(56.0)$ & $349(54.6)$ & $369(57.5)$ \\
\hline & $\mathrm{T} 3$ & $118(6.5)$ & $46(7.0)$ & $35(7.7)$ & $41(5.2)$ & $32(5.0)$ & $37(5.8)$ \\
\hline & $\mathrm{T} 4$ & $27(1.5)$ & $7(1.1)$ & $8(1.8)$ & $13(1.6)$ & $14(2.2)$ & $14(2.2)$ \\
\hline & Unknown & $6(0.3)$ & $1(0.2)$ & 0 & 0 & $1(0.2)$ & $1(0.2)$ \\
\hline & $P$ value & $0.056^{*}$ & $0.135^{* *}$ & & $0.683^{*}$ & $0.819^{* *}$ & \\
\hline \multirow[t]{6}{*}{ N (\%) } & No & $1008(55.7)$ & $364(55.1)$ & $228(50.3)$ & $446(56.0)$ & 328 (51.3) & $330(1.4)$ \\
\hline & N1 & $432(23.9)$ & 158 (23.9) & $100(22.1)$ & 155 (19.5) & $144(22.5)$ & 137 (21.3) \\
\hline & N2 & 185 (10.2) & 77 (11.6) & 66 (14.6) & 85 (10.7) & 91 (14.2) & $82(12.8)$ \\
\hline & N3 & $173(9.6)$ & $60(9.1)$ & $56(12.4)$ & 100 (12.6) & 73 (11.4) & 88 (13.7) \\
\hline & Unknown & $11(0.6)$ & $2(0.3)$ & $3(0.7)$ & $10(1.3)$ & $3(0.5)$ & $5(0.8)$ \\
\hline & $P$ value & $0.022^{*}$ & $0.143^{\star *}$ & & $0.343^{*}$ & $0.638^{* *}$ & \\
\hline \multirow[t]{4}{*}{ ER status (\%) } & Negative & 939 (51.9) & $361(54.6)$ & $237(52.3)$ & 440 (55.3) & 331 (51.8) & $313(48.8)$ \\
\hline & Positive & 659 (36.4) & $241(36.5)$ & 178 (39.3) & $286(35.9)$ & 254 (39.7) & $275(42.8)$ \\
\hline & Unknown & $211(11.7)$ & $59(8.9)$ & $38(8.4)$ & $70(8.8)$ & $54(8.5)$ & $54(8.4)$ \\
\hline & $P$ value & $0.543^{*}$ & $0.363^{\star *}$ & & $0.007^{*}$ & $0.249^{* *}$ & \\
\hline \multirow[t]{4}{*}{ PR status (\%) } & Negative & 935 (51.7) & $344(52.0)$ & $234(51.7)$ & $476(59.8)$ & $364(57.0)$ & 361 (56.2) \\
\hline & Positive & 659 (36.4) & $256(38.7)$ & 179 (39.5) & $247(31.0)$ & $221(34.6)$ & $227(35.4)$ \\
\hline & Unknown & 215 (11.9) & $61(9.2)$ & $40(8.8)$ & $73(9.2)$ & $54(8.5)$ & $54(8.4)$ \\
\hline & $P$ value & $0.463^{*}$ & $0.831^{* *}$ & & $0.096^{*}$ & $0.771^{* * *}$ & \\
\hline \multirow[t]{4}{*}{ NBR (\%) } & 1 & 223 (12.3) & $71(10.7)$ & $67(14.8)$ & 92 (11.6) & $70(11.0)$ & 89 (13.9) \\
\hline & 2,3 & 802 (44.3) & $321(48.6)$ & $241(53.2)$ & 389 (48.9) & 341 (53.4) & $354(55.2)$ \\
\hline & Unknown & 784 (43.3) & $269(40.7)$ & $145(32.0)$ & 315 (39.6) & 228 (35.7) & $199(31.0)$ \\
\hline & $P$ value & $<0.0001^{*}$ & $0.015^{* *}$ & & $<0.0001^{*}$ & $0.212^{* *}$ & \\
\hline \multirow[t]{4}{*}{ HER2 status (\%) } & Negative & $666(36.8)$ & $262(39.6)$ & $181(40.0)$ & $288(36.2)$ & $245(38.3)$ & $246(38.3)$ \\
\hline & Positive & $251(13.9)$ & $70(10.6)$ & $63(13.9)$ & $125(15.7)$ & $96(15.0)$ & $97(15.1)$ \\
\hline & Unknown & $892(49.3)$ & $329(49.8)$ & $210(47.1)$ & $383(48.1)$ & $298(46.6)$ & $299(46.6)$ \\
\hline & $P$ value & $0.628^{*}$ & $0.183^{* *}$ & & $0.550^{*}$ & $0.970^{* *}$ & \\
\hline \multirow[t]{3}{*}{ Adjuvant chemotherapy (\%) } & No & $336(18.6)$ & 118 (17.9) & $59(13.0)$ & $254(31.9)$ & $216(33.8)$ & $206(32.1)$ \\
\hline & Yes & $1473(81.4)$ & $543(82.1)$ & $394(87.0)$ & $542(68.1)$ & $423(66.2)$ & $436(67.9)$ \\
\hline & $P$ value & $0.005^{*}$ & $0.030^{* *}$ & & $0.943^{*}$ & $0.514^{* *}$ & \\
\hline \multirow[t]{4}{*}{ Hormonal therapy (\%) } & No & $1043(57.7)$ & $393(59.5)$ & $260(57.4)$ & $395(49.6)$ & $303(47.4)$ & $303(47.2)$ \\
\hline & Yes & $694(38.4)$ & $243(36.8)$ & $178(39.3)$ & $371(46.6)$ & $323(50.5)$ & $314(48.9)$ \\
\hline & Unknown & $72(4.0)$ & $25(3.8)$ & $15(3.3)$ & $30(3.8)$ & $13(2.0)$ & $25(3.9)$ \\
\hline & $P$ value & $0.776^{*}$ & $0.665^{\star *}$ & & $0.657^{*}$ & $0.142^{\star *}$ & \\
\hline
\end{tabular}

Abbreviations: NBR: nuclear grade;

${ }^{*} P$ value for the comparison of the $\mathrm{BMI}<24$ group and $\mathrm{BMI} \geq 27$ group;

${ }^{*} P$ value for the comparison of the $24 \leq \mathrm{BMI}<27$ group and $\mathrm{BMI} \geq 27$ group. 
A

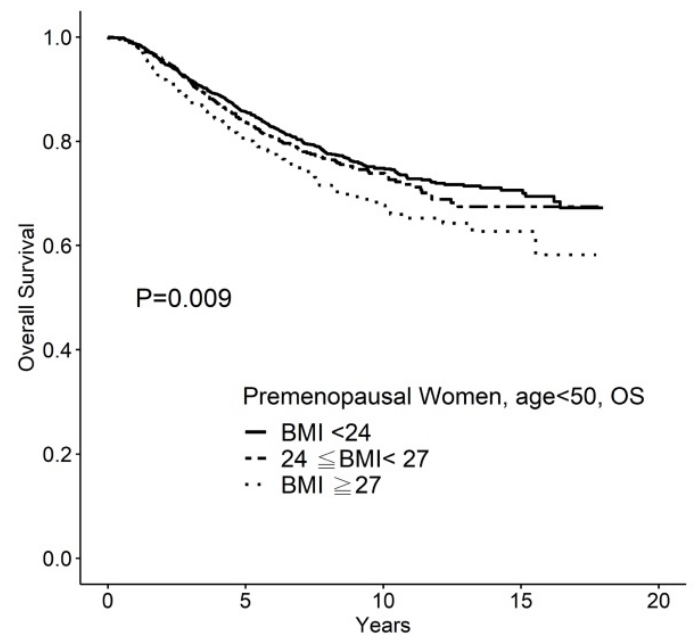

C

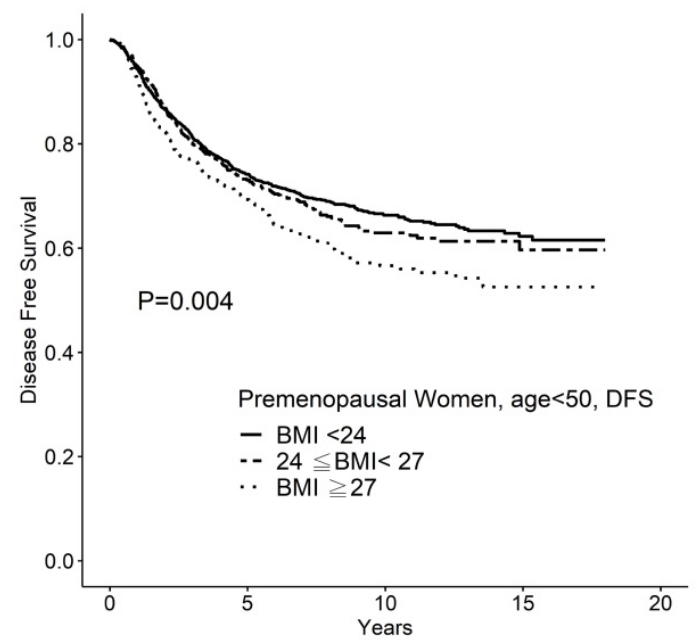

B

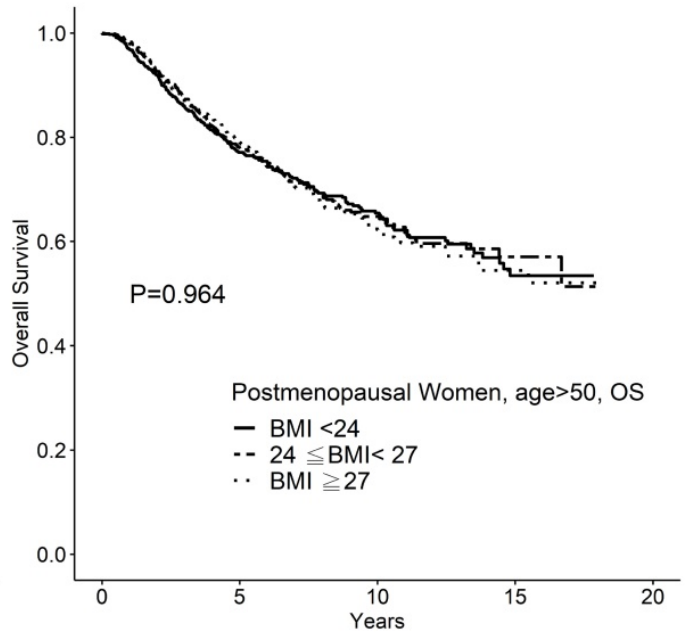

D

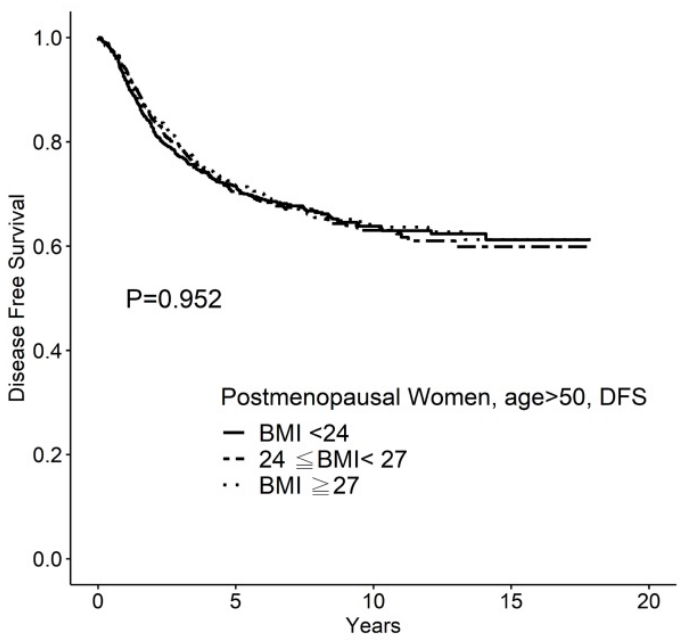

Figure 2. A. Overall survival of breast cancer patients $<50$ years of age. B. Overall survival of breast cancer patients aged $\geq 50$ years. C. Disease-free survival of breast cancer patients $<50$ years of age. D. Disease-free survival of breast cancer patients aged $\geq 50$ years.

Table 2. Multivariate Cox Regression Analysis for the OS and DFS of breast cancer patients

\begin{tabular}{lllllll}
\hline & DFS & \multicolumn{5}{l}{ OS } \\
\cline { 2 - 7 } & $\begin{array}{l}\text { Hazards } \\
\text { Ratio }\end{array}$ & $95 \%$ CI & $P$ value & $\begin{array}{l}\text { Hazards } \\
\text { Ratio }\end{array}$ & $95 \%$ CI & $P$ value \\
\hline All patients & & & 0.569 & & & 0.015 \\
BMI $<24$ & 1.000 & & & 1.000 & & \\
$24 \leq$ BMI $<27$ & 1.019 & $0.910-1.142$ & 0.740 & 1.136 & $1.011-1.277$ & 0.033 \\
BMI $\geq 27$ & 1.046 & $0.929-1.178$ & 0.454 & 1.174 & $1.039-1.326$ & 0.010 \\
Tumor size & & & $<0.0001$ & & & $<0.0001$ \\
T1 & 1.000 & & & 1.000 & & \\
T2 & 1.402 & $1.254-1.567$ & $<0.0001$ & 1.515 & $1.347-1.705$ & $<0.0001$ \\
T3 & 2.011 & $1.681-2.406$ & $<0.0001$ & 2.410 & $2.011-2.889$ & $<0.0001$ \\
T4 & 4.151 & $3.177-5.425$ & $<0.0001$ & 4.881 & $3.749-6.354$ & $<0.0001$ \\
Nodal status & & & $<0.0001$ & & & $<0.0001$ \\
N0 & 1.000 & & & 1.000 & & \\
N1 & 2.436 & $2.148-2.763$ & $<0.0001$ & 2.057 & $1.802-2.348$ & $<0.0001$ \\
N2 & 3.569 & $3.100-4.109$ & $<0.0001$ & 3.224 & $2.791-3.724$ & $<0.0001$ \\
N3 & 5.818 & $5.067-6.681$ & $<0.0001$ & 5.422 & $4.712-6.239$ & $<0.0001$ \\
NBR & & & 0.001 & & & $<0.0001$ \\
1 & 1.000 & & & 1.000 & & \\
2,3 & 1.350 & $1.130-1.613$ & 0.001 & 1.345 & $1.117-1.620$ & 0.002 \\
Unknown & 1.159 & $0.964-1.394$ & 0.117 & 1.103 & $0.910-1.337$ & 0.319 \\
ER status & & & $<0.0001$ & & & $<0.0001$ \\
Positive & 1.000 & & & 1.000 & & \\
Negative & 1.268 & $1.143-1.407$ & $<0.0001$ & 1.295 & $1.162-1.443$ & $<0.0001$ \\
\hline
\end{tabular}

\begin{tabular}{lllllll}
\hline & DFS & \multicolumn{5}{l}{ OS } \\
\cline { 2 - 7 } & $\begin{array}{l}\text { Hazards } \\
\text { Ratio }\end{array}$ & $95 \%$ CI & $P$ value & $\begin{array}{l}\text { Hazards } \\
\text { Ratio }\end{array}$ & $95 \%$ CI & $P$ value \\
\hline $\begin{array}{l}\text { Unknown } \\
\text { HER2 status }\end{array}$ & 1.314 & $1.096-1.576$ & 0.003 & 1.311 & $1.093-1.573$ & 0.003 \\
$\begin{array}{l}\text { Negative } \\
\text { Positive }\end{array}$ & 1.000 & & 0.018 & & & $<0.0001$ \\
Unknown & 1.137 & $0.983-1.315$ & 0.083 & 1.273 & $1.094-1.482$ & 0.002 \\
\hline
\end{tabular}

The prognostic value of intrinsic subtypes is an interesting issue to be studied. We sorted 2758 patients whose HER2 data was available and classified into hormone receptor (HR) positive/HER2 negative $(n=1196)$; HER2 positive (either HER2 IHC $3+$ or FISH positive) $(n=769)$; and triple negative (TNBC) $(\mathrm{n}=793)$ subtypes. We compared the difference of survivals using BMI 27 as cutoff on each subtypes. We found that $\mathrm{BMI} \geq 27$ remained a statistically significant factor for poor disease free survival $(p=0.01)$ and overall survivals for TNBC $(p=0.009)$ breast cancer patients $<50$ years of age, but not elder patients (Table 5). 
Table 3. Multivariate Cox Regression Analysis for OS and DFS of Breast Cancer Patients <50Years of Age

\begin{tabular}{|c|c|c|c|c|c|c|}
\hline & DFS & & & OS & & \\
\hline & $\begin{array}{l}\text { Hazards } \\
\text { Ratio }\end{array}$ & $95 \% \mathrm{CI}$ & $P$ value & $\begin{array}{l}\text { Hazards } \\
\text { Ratio }\end{array}$ & $95 \% \mathrm{CI}$ & $P$ value \\
\hline All patients & & & 0.038 & & & 0.048 \\
\hline BMI <24 & 1.000 & & & 1.000 & & \\
\hline $24 \leq \mathrm{BMI}<27$ & 1.063 & $0.913-1.237$ & 0.432 & 1.175 & $0.996-1.386$ & 0.056 \\
\hline $\mathrm{BMI} \geq 27$ & 1.239 & $1.051-1.460$ & 0.011 & 1.208 & $1.004-1.453$ & 0.045 \\
\hline Tumor size & & & $<0.0001$ & & & $<0.0001$ \\
\hline $\mathrm{T} 1$ & 1.000 & & & 1.000 & & \\
\hline $\mathrm{T} 2$ & 1.370 & $1.187-1.581$ & $<0.0001$ & 1.648 & $1.393-1.949$ & $<0.0001$ \\
\hline T3 & 1.987 & $1.587-2.488$ & $<0.0001$ & 2.779 & 2.181-3.539 & $<0.0001$ \\
\hline $\mathrm{T} 4$ & 3.759 & $2.579-5.480$ & $<0.0001$ & 4.566 & $3.099-6.726$ & $<0.0001$ \\
\hline Nodal status & & & $<0.0001$ & & & $<0.0001$ \\
\hline N0 & 1.000 & & & 1.000 & & \\
\hline N1 & 2.290 & $1.951-2.688$ & $<0.0001$ & 2.181 & $1.816-2.619$ & $<0.0001$ \\
\hline N2 & 3.341 & $2.780-4.014$ & $<0.0001$ & 3.431 & 2.804-4.199 & $<0.0001$ \\
\hline N3 & 4.904 & 4.073-5.904 & $<0.0001$ & 5.001 & $4.087-6.120$ & $<0.0001$ \\
\hline NBR & & & $<0.0001$ & & & $<0.0001$ \\
\hline 1 & 1.000 & & & 1.000 & & \\
\hline 2,3 & 1.477 & $1.176-1.856$ & 0.001 & 1.703 & $1.300-2.231$ & $<0.0001$ \\
\hline Unknown & 1.187 & $0.938-1.503$ & 1.531 & 1.234 & $0.933-1.632$ & 0.141 \\
\hline ER status & & & 0.001 & & & $<0.0001$ \\
\hline Positive & 1.000 & & & 1.000 & & \\
\hline Negative & 1.258 & $1.098-1.441$ & 0.001 & 1.438 & $1.233-1.678$ & $<0.0001$ \\
\hline Unknown & 1.389 & $1.105-1.747$ & 0.005 & 1.505 & $1.170-1.935$ & 0.001 \\
\hline HER2 status & & & 0.207 & & & 0.003 \\
\hline Negative & 1.000 & & & 1.000 & & \\
\hline Positive & 1.075 & $0.886-1.305$ & 0.464 & 1.184 & 0.953-1.469 & 0.127 \\
\hline Unknown & 1.138 & $0.986-1.314$ & 0.076 & 1.323 & $1.125-1.555$ & 0.001 \\
\hline
\end{tabular}

Table 4. Ten-year Disease Free Survival rate and Overall Survival rate of breast cancer patients $<50$ years of age

\begin{tabular}{|c|c|c|c|c|c|c|c|c|}
\hline & \multicolumn{4}{|c|}{ DFS (\%) } & \multicolumn{4}{|c|}{ OS (\%) } \\
\hline & \multicolumn{4}{|c|}{ BMI $\left(\mathrm{kg} / \mathrm{m}^{2}\right)$} & \multicolumn{4}{|c|}{ BMI $\left(\mathrm{kg} / \mathrm{m}^{2}\right)$} \\
\hline & $<24$ & $24 \leq \mathrm{BMI}<27$ & $\geq 27$ & $P$ value & $<24$ & $24 \leq \mathrm{BMI}<27$ & $\geq 27$ & $P$ value \\
\hline \multicolumn{9}{|c|}{ Tumor size } \\
\hline T0-1 & 77.1 & 71.4 & 59.9 & 0.222 & 86.6 & 82.9 & 83.2 & 0.371 \\
\hline T2-4 & 59.9 & 59.2 & 54.1 & 0.071 & 62.7 & 66.1 & 62.0 & 0.058 \\
\hline \multicolumn{9}{|c|}{ Nodal status } \\
\hline Negative & 81.2 & 79.6 & 77.5 & 0.361 & 86.0 & 85.2 & 84.2 & 0.633 \\
\hline Positive & 50.1 & 47.4 & 41.7 & 0.049 & 61.9 & 58.0 & 53.8 & 0.045 \\
\hline \multicolumn{9}{|l|}{ NBR } \\
\hline 1 & 75.1 & 75.2 & 89.4 & 0.965 & 85.2 & 85.3 & 82.6 & 0.840 \\
\hline 2,3 & 61.1 & 56.6 & 49.0 & 0.002 & 68.8 & 64.8 & 60.7 & 0.018 \\
\hline Unknown & 70.9 & 72.8 & 70.7 & 0.563 & 79.5 & 79.3 & 77.0 & 0.807 \\
\hline \multicolumn{9}{|c|}{ ER status } \\
\hline Negative & 56.9 & 64.1 & 52.3 & 0.001 & 72.6 & 69.9 & 62.0 & 0.001 \\
\hline Positive & 69.0 & 65.5 & 69.8 & 0.676 & 79.5 & 74.8 & 78.5 & 0.389 \\
\hline Unknown & 66.1 & 70.6 & 62.7 & 0.538 & 75.3 & 83.1 & 71.1 & 0.386 \\
\hline \multicolumn{9}{|c|}{ HER2 status } \\
\hline Negative & 66.9 & 69.2 & 56.2 & 0.003 & 78.9 & 75.3 & 72.3 & 0.075 \\
\hline Positive & 64.9 & 53.0 & 51.9 & 0.081 & 69.8 & 67.4 & 62.9 & 0.384 \\
\hline Unknown & 67.9 & 64.8 & 65.8 & 0.507 & 74.7 & 72.6 & 68.9 & 0.188 \\
\hline
\end{tabular}

Table 5. Ten-year Disease Free Survival rate and Overall Survival rate of breast cancer among breast cancer subtypes $(\mathrm{N}=2758)$

\begin{tabular}{|c|c|c|c|c|c|c|}
\hline & \multicolumn{3}{|c|}{ Disease free survival (\%) } & \multicolumn{3}{|c|}{ Overall survival (\%) } \\
\hline & All & Age $\leq 50$ & Age $>50$ & All & Age $\leq 50$ & Age $>50$ \\
\hline BMI & $\geq 27<27$ & $\geq 27<27$ & $\geq 27<27$ & $\geq 27<27$ & $\geq 27<27$ & $\geq 27<27$ \\
\hline HR+/HER2- & 52.955 .9 & $45.0 \quad 57.2$ & $51.7 \quad 61.7$ & 56.373 .4 & $71.4 \quad 77.1$ & $44.4 \quad 66.6$ \\
\hline$P$ value & 0.507 & 0.304 & 0.875 & 0.414 & 0.616 & 0.875 \\
\hline HER 2+ & $48.0 \quad 58.7$ & 37.458 .3 & 59.759 .8 & $53.0 \quad 57.0$ & $52.5 \quad 61.1$ & $55.2 \quad 49.0$ \\
\hline$P$ value & 0.204 & 0.029 & 0.810 & 0.211 & 0.265 & 0.643 \\
\hline TNBC & 42.656 .4 & 37.257 .8 & $43.3 \quad 54.4$ & $56.3 \quad 68.3$ & $56.6 \quad 72.9$ & 59.961 .0 \\
\hline$P$ value & 0.010 & $<0.001$ & 0.732 & 0.009 & $<0.001$ & 0.845 \\
\hline
\end{tabular}

*HR: hormone receptor; HER2: human epidermal growth factor receptor 2; TNBC: triple negative breast cancer.

\section{Discussion}

The present study reveal that BMI at diagnosis, is an independent prognostic factor for survival in premenopausal Asian breast cancer patients. We found that higher-BMI patients present mostly with larger tumor sizes, advanced stage, higher nuclear grades, and higher percentages of ER positivity. A BMI $\geq 27$ is statistically associated with poor OS but doesn't significantly affect DFS. The impact of BMI on OS affects only on patients aged $<50$ but not $\geq 50$ years old. In patients aged $<50$ years, a BMI $\geq 27$ is an independent prognostic factor for both DFS and OS, after adjusting for tumor size, nodal status, ER status, and HER2 status. Our study suggests that the effect of obesity is context-dependent with regard to age, and menopausal status.

We and other studies have shown that obese breast cancer patients tend to have an advanced stage, higher nuclear grade, but more HR positive tumors $[18,24,26]$. The plausible mechanisms for the association of high tumor size, stage; and nuclear grade with obese breast cancer patients were multifactorial [24]. One of the explanations for the association with advanced stage, or tumor size was the delayed diagnosis due to obesity [26]. Biological mechanism such as the insulin resistance/insulin like growth factor-1 axis, enriched humoral factors such as adipokines, pro-inflammatory cytokines have been addressed [39-41]. The reports on the association of breast cancer subtypes with obesity were inconsistent. Majority of the reports did not find a positive correlation with any subtypes of breast cancer with obesity, whereas some reported increased ER positive cancers on obese patients [26, 28]. As estrogen is a promotor for breast cancer progression, our data showed that upon subgroup analysis, the association of increased ER positive breast cancers was only on the post-menopausal subgroup. This feature suggests that increased estrogen level on post-menopausal obese women is a possible explanation. Our whole cohort analysis suggested that HER2 negativity had a marginal positive correlation with higher BMI. However, the association did not exist on the subsequent subset analysis, regardless the age, or BMI categories. Therefore, the association of HER2 negativity with obesity on our dataset was inconclusive.

A meta-analysis conducted by Protani et al. analyzed 43 relevant studies published between 1960 and 1990, in which obesity was measured by either BMI or waist-hip ratios. The conclusion was that obesity was modestly associated with poor overall and breast cancer-specific survival [20]. However, the study period spanning over three decades, the reported studies might not have the same quality of 
treatments. Jiralerspong et al in another metanalysis, using more updated data, also concluded similar results. [42] Several large scale population-based studies have been addressed the influence of obesity on both OS and DFS. Majed et al. used a BMI of 30 as a cutoff value and followed a breast cancer cohort for up to 20 years. The authors found that obesity was associated with shorter distant metastasis-free and secondary malignancy-free times. Disease-free intervals and survival were reduced among obese patients [21]. A Danish study enrolled more than 50,000 patients who were diagnosed between 1977 and 2006; BMI information was available for only approximately 1,900 patients who received chemotherapy. That could have a bias toward advanced, and HR negative population. The times to distant metastasis and breast cancer-related death, were significantly worse among patients with BMI $>30$, compared to those with BMI <25 [19]. Ligibel et al. did a post hoc analysis on CALGB 9741 study and found that BMI is an independent prognostic factors for survivals on patients receiving optimal dose adjuvant chemotherapy [43]. In summary, in unselected population or chemotherapy-treated population, obesity was a prognostic factors for breast cancer survivals.

The differently weighted values of obesity with regard to menopausal status have been addressed as well. Loi et al. reviewed 10 different studies on premenopausal or postmenopausal patients with varied BMI category definitions. Although they concluded that obesity was an independent prognostic factors for both groups, the HR ratio was even greater on the pre-menopausal group [31]. Whiteman divided approximately 2,500 breast cancer patients into four groups according to BMI, and found a significant trend toward an increased death rate in obese peri- and premenopausal women, but not in obese postmenopausal women [23]. Berclaz et al. analyzed an International Breast Cancer Study Group (IBSCG) trial cohort of patients and showed that decreases in DFS and OS among obese (BMI>30) patients were limited to pre- and perimenopausal patients [32]. Study from Protani et al also showed similar trends that the effect of obesity on survival was more significant in premenopausal women [20]. Kim, et al., however did an analysis from Korean on stage I-III patients and concluded a contradictory results that obesity was associated with negative impact of relapse free survival only in HR positive and postmenopausal groups [44].

The inconsistent data derived from these studies might be due to relatively small sample sizes or inadequate follow-up periods. Our study cohort was relative large $(\mathrm{N}=5,000)$ and was the longest follow-up period up to 20 years. The results generated from our study are comparable with the majority of the studies that suggest that obese women with poor survival rates are mainly premenopausal (age $<50$ years) women. The dichotomy of the effect of obesity on breast cancer is interesting. Obesity increases the breast cancer risk in postmenopausal women, but is inversely related to the risk in premenopausal women [45]. On the basis of the results from our study and others, obesity seems to be associated with poor prognosis in premenopausal women, but not in postmenopausal women. To date, the underlying mechanism of this finding is unclear, and mostly hypothetical [41, 46, 47]. Since breast cancer is a sex hormone-associated malignancy, the role of sex hormones has been discussed at length, even in the Chinese population [48]. Possible explanations included: advanced stage at diagnosis, increased paracrine sex hormones in the surrounding adipose tissue [49], attenuated post-chemotherapy amenorrhea effects in obese women, and lower levels of circulating estradiol in premenopausal women who were more sensitive to high-ER tumor status. Another hypothesis for the higher risk of recurrence in obese premenopausal women is that non-sex hormonal mechanisms might play a role $[39,50]$. Our subgroup analysis showed that the effect of obesity on prognosis was more significant in ER-negative patients or TNBC subset, suggesting that an ER-independent pathway might be involved. For example, leptin, a growth factor for malignant breast cells, might play a role in the mechanism underlying the association between BMI and breast cancer [40, 51]. Obesity might be associated with a untoward immune microenvironment for host immunity against breast cancer [52]. However, no causal relationships have been confirmed. We also noted that obese patients were increased rate of positive node status, and would increase recurrence and mortality. According to our treatment guidelines, the group of patients should receive adjuvant chemotherapy and radiotherapy if they present with pathological $\mathrm{T} 3$ or N2 disease. The GEICAM study group analyzed pooled data from three different adjuvant chemotherapy clinical trials and found that severe obesity is a poor prognostic factor across all subtypes of breast cancers [53]. A Mexican study recently found that obesity is an independent poor prognostic factor for locally advanced breast cancer receiving neoadjuvant chemotherapy [54]. The causal relationship is unknown; whether it could be attributed to suboptimal chemotherapy for obese patients is not clear.

The limitation of our study is that these data were collected from a single institute and, thus, might 
not adequately represent the overall Asian population. The study lacks cancer-specific survival data, and there was no difference in disease-free survival on whole cohort, which might be biased by confounding factors. However, since Asian women tend to be much thinner than Caucasian women, a cutoff BMI value of 27 could be applicable for this population [33, 35, 55]. In contrast to populationbased studies, our patients received guidelineoriented therapy and regular follow-up at single institution. Our patients included all stage I to III breast cancer patients who received primary surgery, regardless of the presence or absence of subsequent adjuvant therapy; therefore, this information is more close to real world data than that of clinical trial-based cohorts. The data generated for this analysis are relatively reliable. Although there is lack of cause-specific survival, the significance of obesity on DFS and OS was confirmed by a multivariate analysis.

In summary, we confirmed that in an Asian population, young obese women had a higher risk of breast cancer recurrence and mortality than lean women. This tendency was more prominent in patients with ER-negative and advanced-stage tumors. We suggest that further investigations of the underlying mechanism and strategies to improve outcomes in this population are warranted.

\section{Acknowledgements}

\section{Ethics}

This work is in accordance with Declaration of Helsinki, and is approved by Institutional Review Board No. 101-1637B.

The authors are grateful to Biostatical Section, Clinical Trial Center, Chang Gung Memorial Hospital (MOHW110-TDU-B-212-123005) for the statistical support.

\section{Author Contributions}

Y-C Lin: Study design, data collection and analysis, manuscript preparation; $\mathrm{H}-\mathrm{H}$ Cheng; data analysis, manuscript preparation; S-C Chen: study design, data collection and analysis; W-C Shen: data analysis; Y-T Huang: data analysis.

\section{Competing Interests}

The authors have declared that no competing interest exists.

\section{References}

1. Hill JO. Understanding and addressing the epidemic of obesity: an energy balance perspective. Endocr Rev 2006; 27: 750-761.

2. Calle EE, Rodriguez C, Walker-Thurmond K, et al. Overweight, obesity, and mortality from cancer in a prospectively studied cohort of U.S. adults. N Engl J Med 2003; 348: 1625-1638.

3. Parekh N, Chandran U, Bandera EV. Obesity in cancer survival. Annu Rev Nutr 2012; 32: 311-342.
4. Renehan AG, Tyson M, Egger M, et al. Body-mass index and incidence of cancer: a systematic review and meta-analysis of prospective observational studies. Lancet 2008; 371: 569-578.

5. La Vecchia C, Giordano SH, Hortobagyi GN, et al. Overweight, obesity, diabetes, and risk of breast cancer: interlocking pieces of the puzzle. Oncologist 2011; 16: 726-729.

6. Rapp K, Schroeder J, Klenk J, et al. Obesity and incidence of cancer: a large cohort study of over 145,000 adults in Austria. Br J Cancer 2005; 93: 1062-1067.

7. McTiernan A, Rajan KB, Tworoger SS, et al. Adiposity and sex hormones in postmenopausal breast cancer survivors. J Clin Oncol 2003; 21: 1961-1966.

8. Anderson GL, Neuhouser ML. Obesity and the risk for premenopausal and postmenopausal breast cancer. Cancer Prev Res (Phila) 2012; 5: 515-521.

9. Nahleh Z. Breast cancer, obesity and hormonal imbalance: a worrisome trend. Expert Rev Anticancer Ther 2011; 11: 817-819.

10. Roberts DL, Dive C, Renehan AG. Biological mechanisms linking obesity and cancer risk: new perspectives. Annu Rev Med 2010; 61: 301-316.

11. Pujol P, Galtier-Dereure F, Bringer J. Obesity and breast cancer risk. Hum Reprod 1997; 12 Suppl 1: 116-125.

12. Gu F, Kraft P, Rice M, Michels KB. Leptin and leptin receptor genes in relation to premenopausal breast cancer incidence and grade in Caucasian women. Breast Cancer Res Treat 2012; 131: 17-25.

13. Wang D, Dubois RN. Associations between obesity and cancer: the role of fatty acid synthase. J Natl Cancer Inst 2012; 104: 343-345.

14. Sung MK, Yeon JY, Park SY, et al. Obesity-induced metabolic stresses in breast and colon cancer. Ann N Y Acad Sci 2011; 1229: 61-68.

15. de Azambuja E, McCaskill-Stevens W, Francis P et al. The effect of body mass index on overall and disease-free survival in node-positive breast cancer patients treated with docetaxel and doxorubicin-containing adjuvant chemotherapy: the experience of the BIG 02-98 trial. Breast Cancer Res Treat 2010; 119: 145-153.

16. Robinson PJ, Bell RJ, Davis SR. Obesity is associated with a poorer prognosis in women with hormone receptor positive breast cancer. Maturitas 2014; 79: 279-286.

17. Nindrea RD, Aryandono T, Lazuardi L, Dwiprahasto I. Association of Overweight and Obesity with Breast Cancer During Premenopausal Period in Asia: A Meta-Analysis. Int J Prev Med 2019; 10: 192.

18. Haakinson DJ, Leeds SG, Dueck AC et al. The impact of obesity on breast cancer: a retrospective review. Ann Surg Oncol 2012; 19: 3012-3018.

19. Ewertz M, Jensen MB, Gunnarsdottir KA et al. Effect of obesity on prognosis after early-stage breast cancer. J Clin Oncol 2011; 29: 25-31.

20. Protani M, Coory M, Martin JH. Effect of obesity on survival of women with breast cancer: systematic review and meta-analysis. Breast Cancer Res Treat 2010; 123: 627-635

21. Majed B, Moreau T, Senouci $K$, et al. Is obesity an independent prognosis factor in woman breast cancer? Breast Cancer Res Treat 2008; 111: 329-342.

22. Caan BJ, Kwan ML, Hartzell G, et al. Pre-diagnosis body mass index, post-diagnosis weight change, and prognosis among women with early stage breast cancer. Cancer Causes Control 2008; 19: 1319-1328.

23. Whiteman MK, Hillis SD, Curtis KM, et al. Body mass and mortality after breast cancer diagnosis. Cancer Epidemiol Biomarkers Prev 2005; 14: 2009-2014.

24. Porter GA, Inglis KM, Wood LA, et al. Effect of obesity on presentation of breast cancer. Ann Surg Oncol 2006; 13: 327-332.

25. Chlebowski RT. Obesity and early-stage breast cancer. J Clin Oncol 2005; 23: 1345-1347.

26. Biglia N, Peano E, Sgandurra P, et al. Body mass index (BMI) and breast cancer: impact on tumor histopathologic features, cancer subtypes and recurrence rate in pre and postmenopausal women. Gynecol Endocrinol 2013; 29. 263-267.

27. Kabore EG, Guenancia C, Vaz-Luis I, et al. Association of body mass index and cardiotoxicity related to anthracyclines and trastuzumab in early breast cancer: French CANTO cohort study. PLoS Med 2019; 16: e1002989.

28. Berclaz G, Li S, Price KN, et al. Body mass index as a prognostic feature in operable breast cancer: the International Breast Cancer Study Group experience. Ann Oncol 2004; 15: 875-884.

29. Conroy SM, Maskarinec G, Wilkens LR, et al. Obesity and breast cancer survival in ethnically diverse postmenopausal women: the Multiethnic Cohort Study. Breast Cancer Res Treat 2011; 129: 565-574.

30. Sestak I, Distler W, Forbes JF, et al. Effect of body mass index on recurrences in tamoxifen and anastrozole treated women: an exploratory analysis from the ATAC trial. J Clin Oncol 2010; 28: 3411-3415.

31. Loi S, Milne RL, Friedlander ML, et al. Obesity and outcomes in premenopausal and postmenopausal breast cancer. Cancer Epidemiol Biomarkers Prev 2005; 14: 1686-1691.

32. Berclaz G, Li S, Price KN, et al. Body mass index as a prognostic feature in operable breast cancer: the International Breast Cancer Study Group experience. Ann Oncol 2004; 15: 875-884.

33. Hwang LC, Bai CH, Sun CA, et al. Prevalence of metabolically healthy obesity and its impacts on incidences of hypertension, diabetes and the metabolic syndrome in Taiwan. Asia Pac J Clin Nutr 2012; 21: 227-233.

34. Hwang LC, Bai CH, Chen CJ. Prevalence of obesity and metabolic syndrome in Taiwan. J Formos Med Assoc 2006; 105: 626-635.

35. Lin YC, Yen LL, Chen SY, et al. Prevalence of overweight and obesity and its associated factors: findings from National Nutrition and Health Survey in Taiwan, 1993-1996. Prev Med 2003; 37: 233-241. 
36. Pan WH, Flegal KM, Chang HY, et al. Body mass index and obesity-related metabolic disorders in Taiwanese and US whites and blacks: implications for definitions of overweight and obesity for Asians. Am J Clin Nutr 2004; 79: 31-39.

37. Chlebowski RT, Chen Z, Anderson GL, et al. Ethnicity and breast cancer: factors influencing differences in incidence and outcome. J Natl Cancer Inst 2005; 97: 439-448.

38. Lutz MP, Zalcberg JR, Ducreux M, et al. Highlights of the EORTC St. Gallen International Expert Consensus on the primary therapy of gastric, gastroesophageal and oesophageal cancer - differential treatment strategies for subtypes of early gastroesophageal cancer. Eur J Cancer 2012; 48: 2941-2953.

39. Goodwin PJ, Ennis M, Pritchard KI, et al. Insulin-like growth factor binding proteins 1 and 3 and breast cancer outcomes. Breast Cancer Res Treat 2002; 74: 65-76.

40. Hu X, Juneja SC, Maihle NJ, et al. Leptin--a growth factor in normal and malignant breast cells and for normal mammary gland development. J Natl Cancer Inst 2002; 94: 1704-1711.

41. Khandekar MJ, Cohen P, Spiegelman BM. Molecular mechanisms of cancer development in obesity. Nat Rev Cancer 2011; 11: 886-895.

42. Jiralerspong S, Goodwin PJ. Obesity and Breast Cancer Prognosis: Evidence, Challenges, and Opportunities. J Clin Oncol 2016; 34: 4203-4216.

43. Ligibel JA, Cirrincione CT, Liu M, et al. Body Mass Index, PAM50 Subtype, and Outcomes in Node-Positive Breast Cancer: CALGB 9741 (Alliance). J Natl Cancer Inst 2015; 107.

44. Kim JY, Lee DW, Lee KH et al. Prognostic role of body mass index is different according to menopausal status and tumor subtype in breast cancer patients. Breast Cancer Res Treat 2019; 176: 453-460.

45. Bianchini F, Kaaks R, Vainio H. Overweight, obesity, and cancer risk. Lancet Oncol 2002; 3: 565-574.

46. Goodwin PJ, Ennis M, Pritchard KI, et al. Fasting insulin and outcome in early-stage breast cancer: results of a prospective cohort study. J Clin Oncol 2002; 20: 42-51.

47. Rose DP, Komninou D, Stephenson GD. Obesity, adipocytokines, and insulin resistance in breast cancer. Obes Rev 2004; 5: 153-165.

48. Yu H, Shu XO, Shi R, et al. Plasma sex steroid hormones and breast cancer risk in Chinese women. Int J Cancer 2003; 105: 92-97.

49. Thijssen JH, Blankenstein MA. Endogenous oestrogens and androgens in normal and malignant endometrial and mammary tissues. Eur J Cancer Clin Oncol 1989; 25: 1953-1959.

50. Gu JW, Young E, Patterson SG, et al. Postmenopausal obesity promotes tumor angiogenesis and breast cancer progression in mice. Cancer Biol Ther 2011; 11: 910-917.

51. Falk RT, Brinton LA, Madigan MP, et al. Interrelationships between serum leptin, IGF-1, IGFBP3, C-peptide and prolactin and breast cancer risk in young women. Breast Cancer Res Treat 2006; 98: 157-165.

52. Naik A, Monjazeb AM, Decock J. The Obesity Paradox in Cancer, Tumor Immunology, and Immunotherapy: Potential Therapeutic Implications in Triple Negative Breast Cancer. Front Immunol 2019; 10: 1940.

53. Pajares B, Pollan M, Martin M, et al. Obesity and survival in operable breast cancer patients treated with adjuvant anthracyclines and taxanes according to pathological subtypes: a pooled analysis. Breast Cancer Res 2013; 15: R105.

54. Arce-Salinas C, Aguilar-Ponce JL, Villarreal-Garza C, et al. Overweight and obesity as poor prognostic factors in locally advanced breast cancer patients. Breast Cancer Res Treat 2014; 146: 183-188.

55. Pan WH, Yeh WT. How to define obesity? Evidence-based multiple action points for public awareness, screening, and treatment: an extension of Asian-Pacific recommendations. Asia Pac J Clin Nutr 2008; 17: 370-374 